\title{
Protoplast Fusion and Gene Recombination in the Uncommon Actinomycete Planobispora rosea Producing GE2270
}

\author{
Fabrizio Beltrametti ${ }^{\dagger}$, Daniele Barucco, Roberta Rossi, Enrico Selva, Flavia Marinelli
}

Received: May 7, 2007 / Accepted: July 17, 2007

(C) Japan Antibiotics Research Association

\begin{abstract}
An efficient method for protoplast generation for the uncommon actinomycete Planobispora rosea, the producer of the thiazolylpeptide antibiotic GE2270, was developed using a combination of hen egg white lysozyme and Streptomyces globisporus mutanolysin. This method converted more than $70 \%$ of vegetative mycelium to protoplasts, which were then regenerated with $50 \%$ efficiency in an optimized medium. When $P$. rosea protoplasts were efficiently fused, recombination between different antibiotic (streptomycin and gentamicin) resistance markers originated sensitive strains $\left(\operatorname{str}^{\mathrm{s}} \mathrm{gen}{ }^{\mathrm{s}}\right)$ at frequencies as high as $18 \%$ and double resistant fusants $\left(\operatorname{str}^{\mathrm{r}} \mathrm{gen}^{\mathrm{r}}\right)$ at frequencies as high as $29 \%$. Double resistant fusants showed GE2270 productivity intermediate between the productivity of the parental strains. Protoplast generation and fusion in $P$. rosea makes whole genome shuffling feasible as an approach to be used alternately with classical random mutagenesis in industrial strain improvement programs.
\end{abstract}

Keywords Planobispora rosea, protoplast, genome shuffling, GE2270, antibiotic, genetic improvement, actinomycetes

\section{Introduction}

Planobispora rosea ATCC 53773 [1] is the producer of the thiazolylpeptide antibiotic GE2270 [2], which acts as a specific inhibitor of bacterial protein synthesis by binding to Elongation Factor Tu. A semi-synthetic derivative of GE2270, named BI-ACNE, has been recently evaluated for clinical development as an anti-acne antibiotic for its activity against Propionibacterium acnes [3]. Planobispora rosea belongs to the so-called group of uncommon or rare actinomycetes, i.e. filamentous actinomycetes other than streptomycetes, which are quite difficult to isolate, cultivate and genetically manipulate [4].

Rare actinomycetes often produce industrially relevant metabolites, but their cost-effective exploitation is usually impeded by the lack of genetic tools, which hinders strain and product improvement. Since mobile genetic elements and conjugation systems are poorly characterized in these microorganisms, a crucial methodology to achieve their transformation by exogenous DNA or to recombine whole genomes (Whole Genome Shuffling-WGS) is based on protoplast manipulation and fusion [5 12]. Protoplast fusion allows the combination of desirable alleles from divergent selection lines into a single strain as a result of the high frequency of intra-strain chromosomal recombination, even when molecular information on the mutated genes is not available. For these reasons, WGS is being increasingly alternated with random mutation cycles (Classical Strain Improvement-CSI) in any breeding program for the improvement of a quantitative character such as antibiotic yield [7, 13 18].

Protoplast preparation and regeneration in Streptomyces spp. was originally reported by Okanishi and co-workers

$\dagger$ Present address: Actygea S.r.1., via R. Lepetit 34, 21040 Gerenzano (VA) Italy
F. Beltrametti (Corresponding author), D. Barucco, R. Rossi, E. Selva: Vicuron Pharmaceuticals S.r.1, via R. Lepetit 34, 21040 Gerenzano (VA) Italy, E-mail: fbeltramett@actygea.it

F. Marinelli: Dipartimento di Biotecnologie e Scienze Molecolari Universita dell'Insubria, Via J. H. Dunant 3, 21100 Varese Italy 
[19]. The method developed for streptomycetes was then applied with uneven success to Micromonospora spp. [20 23], Brevibacillus spp. [8], Amycolatopsis spp. [24, 25], Actinoplanes spp. [26, 27], Saccharopolyspora erythraea [28], Actinomadura verrucosa [29] and Kibdelosporangium aridum [27]. A general conclusion from these studies is that the protocol to be used is species or even strain specific. In other industrially valuable actinomycetes, ad hoc techniques have been developed [9] in some cases, but methods with broad applicability and robustness that can be applied for WGS in strain improvement programs are not currently available.

In this report, we describe our methodology for preparing Planobispora rosea protoplasts with high efficiency and regenerating these protoplasts so they resume normal filamentous growth. This methodology enabled protoplast fusion to be used to recombine antibiotic resistant genes previously selected in different GE2270 high-producing mutants $[30,31]$.

\section{Materials and Methods}

\section{Strains and Cultural Conditions}

Planobispora rosea PR1/5 is a high producing variant induced by MNNG treatment [30, 31]. Strain 162 is a spontaneous PR1/5 streptomycin resistant derivative $\left(\operatorname{str}^{\mathrm{r}}\right)$; strain RS9 is a spontaneous PR1/5 streptomycin and rifamycin resistant $\left(\operatorname{str}^{\mathrm{r}}\right.$ rif $\left.^{\mathrm{r}}\right)$ derivative; strains G2 and G67 are spontaneous PR1/5 gentamicin resistant $\left(\mathrm{gen}^{\mathrm{r}}\right)$ derivatives [30]. Strain characteristics are summarized in Table 1.

Strains were maintained as a lyophilised Master Cell Bank (MCB). A Working Cell Bank (WCB) was prepared from the first generation slant originating from the $\mathrm{MCB}$ as already described [32]. Cryo-vials from the WCB were thawed at room temperature and $2 \mathrm{ml}$ were used to inoculate $100 \mathrm{ml}$ of Medium V in 500-ml baffled flasks. Strains were grown for $72 \sim 96$ hours on a rotary shaker at
$200 \mathrm{rpm}$ and $30^{\circ} \mathrm{C}$. For protoplast preparation, $10 \%$ of the culture was inoculated in $100 \mathrm{ml}$ of Medium VM and growth was allowed for further 96 hours at $30^{\circ} \mathrm{C}$ and at $200 \mathrm{rpm}$. Medium V0.1 was routinely used as solid medium. Media composition is described in Table 2.

\section{Protoplasts Formation, Fusion and Regeneration}

Dispersed vegetative mycelium, prepared as above described, was centrifuged at $3250 \times g$, washed once in $\mathrm{P}$ medium [19] and suspended into an equal volume of P medium. Lysozyme, achromopeptidase, proteinase, endoprotease and mutanolysin (SIGMA) for cell wall digestion were dissolved in $\mathrm{P}$ medium and added at the final concentrations reported in Table 3. The non-ionic detergent Pluronic (SIGMA) was added at the final concentration of $100 \mathrm{mg} /$ liter. After 16 24 hours incubation with reciprocal shaking, protoplasts were detached from residual mycelium clumps by thoroughly pipetting up and down. Protoplasts were then separated from residual hyphal fragments by filtration through glass wool and eventually through $5 \mu \mathrm{m}$ durapore membrane filters (MILLIPORE), then centrifuged at $30000 \times g$, and re-suspended in fresh $\mathrm{P}$ medium. Formation of protoplasts was followed by microscopic observation and they were counted by using a Petroff-Hausser counting chamber and a Zeiss phase-contrast microscope at $400 \times$.

Fusion of protoplasts was performed essentially as suggested by Hopwood et al. [12]. In brief, $10^{7}$ protoplasts for each strain involved in the fusion, were pooled, collected by centrifugation and suspended in $0.5 \mathrm{ml}$ of $40 \%$ PEG1000 (SIGMA) dissolved in P medium. After $1 \sim 3$ minutes incubation at room temperature, PEG1000 was diluted to $10 \%$ with the addition of 3 volumes of $\mathrm{P}$ medium. The protoplast mixture was centrifuged at $10000 \times g$, decanted and then re-suspended in fresh $\mathrm{P}$ medium.

Regeneration of protoplasts was performed using the overlay technique suggested by Shirahama et al. [33]. Plates were seeded by pouring $0.2 \mathrm{ml}$ of protoplast

Table 1 Strains used in this study

\begin{tabular}{|c|c|}
\hline Strain & Relevant phenotype \\
\hline Planobispora rosea PR1/5* & $\begin{array}{l}\text { High producing variant of the thiazolylpeptide GE2270, isolated by } \\
\text { selection from the parental ATCC } 53773 \text { after MNNG treatment }\end{array}$ \\
\hline P. rosea $162^{*}$ & str $^{r}$, high GE2270 producer, isolated from PR1/5 by selection on $200 \mu \mathrm{g} / \mathrm{ml}$ streptomycin \\
\hline P. rosea $\mathrm{G} 67^{*}$ & gen $^{r}$, high GE2270 producer, isolated from PR1/5 by selection on $1 \mu \mathrm{g} / \mathrm{ml}$ gentamicin \\
\hline P. rosea G2* & gen $^{r}$, high GE2270 producer, isolated from PR1/5 by selection on $1 \mu \mathrm{g} / \mathrm{ml}$ gentamicin \\
\hline P. rosea $\mathrm{RS9}^{*}$ & str $^{r}$, rifr, high GE2270 producer, isolated from 162 by selection on $100 \mu \mathrm{g} / \mathrm{ml}$ rifamycin \\
\hline
\end{tabular}

* Reference 30. 
Table 2 Medium composition

\begin{tabular}{|c|c|c|c|c|c|}
\hline Component & Medium V & Medium VM & Medium V0.1 & M3 & Medium VMS0.1 \\
\hline Soluble starch (DIFCO) (g/liter) & 24 & 24 & 2.4 & 2.4 & 2.4 \\
\hline Dextrose (g/liter) & 1 & 1 & 0.1 & 0.1 & 0.1 \\
\hline Meat extract (g/liter) & 3 & 3 & 0.3 & 0.3 & 0.3 \\
\hline Yeast extract (g/liter) & 5 & 5 & 0.5 & 0.5 & 0.5 \\
\hline Triptose (g/liter) & 5 & 5 & 0.5 & 0.5 & 0.5 \\
\hline Agar (g/liter) & 0 & 0 & 15 & 15 & 0 \\
\hline Low melting point agarose (g/liter) & 0 & 0 & 0 & 0 & 4 \\
\hline L-Proline (g/liter) & 0 & 3.5 & 0 & 3.5 & 3.5 \\
\hline Sucrose (g/liter) & 0 & 103 & 0 & 80 & 103 \\
\hline $\mathrm{MgCl}_{2}$ (mmol/liter) & 0 & 0 & 0 & 10 & 0 \\
\hline $\mathrm{CaCl}_{2}$ (mmol/liter) & 0 & 0 & 0 & 50 & 0 \\
\hline
\end{tabular}

Media were prepared in de-ionized water and $\mathrm{pH}$ was adjusted to 7.2 after sterilization.

suspension on M3 agar and then overlaid with $4.0 \mathrm{ml}$ of melted, pre-cooled (about $30^{\circ} \mathrm{C}$ ) Medium VMS0.1 (Table 2). Plates were incubated at $28^{\circ} \mathrm{C}$ for $20 \sim 30$ days. Since protoplasts hardly regenerated on media supplemented with antibiotics, the efficiency of recombination among antibiotic resistant strains was determined by replicaplating the regenerated colonies onto Medium V0.1 plates and onto Medium V0.1 plates supplemented with $200 \mathrm{mg} /$ liter streptomycin, $100 \mathrm{mg} / \mathrm{liter}$ rifamycin or $1.0 \mathrm{mg} /$ liter gentamicin.

\section{Liquid Media Fermentation}

For antibiotic production, growth conditions in liquid media were essentially as described by Gastaldo and Marinelli [31]. Strains were inoculated in $100 \mathrm{ml}$ of D/Seed vegetative medium in 500-ml baffled flasks. After 72-hour growth on a rotary shaker at $200 \mathrm{rpm}$ and $28^{\circ} \mathrm{C}, 10 \%$ of the culture was inoculated in $100 \mathrm{ml}$ of Medium $\mathrm{C}$ and fermentation was allowed for 7 days at $28^{\circ} \mathrm{C}$ and $200 \mathrm{rpm}$. Production was estimated by HPLC as described below and productivities reported are relative to the PR1/5 strain.

\section{GE2270 Extraction and Analysis}

Samples were collected from fermentation flasks and processed for GE2270 extraction by mixing one volume of whole culture with two volumes of acetonitrile and vortexing at room temperature for 30 seconds. Samples were centrifuged at $3250 \times g$ for 10 minutes and the supernatant was analysed by HPLC using an analytical $5 \mu \mathrm{m}$ particle size Ultrasphere ODS (Beckman) column $(4.6 \times 250 \mathrm{~mm})$ eluted at $1.5 \mathrm{ml} / \mathrm{minute}$ flow rate with a 20 minutes linear gradient from $45 \%$ to $75 \%(\mathrm{v} / \mathrm{v})$ of Phase B. Phase A was $20 \mathrm{mmol} /$ liter $\mathrm{NaH}_{2} \mathrm{PO}_{4}: \mathrm{CH}_{3} \mathrm{CN} 9: 1$ (v/v) and Phase $\mathrm{B}$ was $20 \mathrm{mmol} /$ liter $\mathrm{NaH}_{2} \mathrm{PO}_{4}: \mathrm{CH}_{3} \mathrm{CN} 3: 7$ (v/v) mixture. The chromatography was performed with a Hewlett Packard model 1100 HPLC system and detection was at $310 \mathrm{~nm}$. As standard for antibiotic titre determination, a sample of GE2270 antibiotic was used. GE2270 production was calculated as already described [31].

\section{Selection Criteria and Characterization of Resistant Mutants}

GE2270 production in the parental populations was estimated by fermenting ca. 50 independent clones in triplicate. Clones were distributed in classes on the bases of their average GE2270 productivity, giving a normal distribution. Production of GE2270 double resistant clones was estimated by fermenting at least 40 independent mutants in triplicate, and showed a peculiar distribution for each combination of the resistance markers. The Standard Deviation (SD) among the replicas did not exceed 10\%. We define as high producers those mutants producing more than the highest GE2270 producing clone among parental controls [30].

\section{Results and Discussion}

\section{Protoplast Preparation}

Reported methods for protoplast production are based on lysozyme treatment combined with other cell wall hydrolysing enzymes [9 12, 27, 33 35]. The application of these protocols to members of Streptomyces gave a vast range of efficiencies in protoplast formation varying within species and strains. When we tried to apply the known procedures to the Planobispora rosea strains described in Table 1, the first obstacle was that none of the described 
media $[9,36]$ designed for generating biomass for protoplast formation, supported Planobispora rosea growth. To overcome this problem, we screened a number of rich media previously used for Planobispora rosea biomass production (Beltrametti et al. unpublished results) and supplemented them with different concentrations of sucrose and proline to acclimate Planobispora rosea to the components of the hyper tonic buffer (P medium) used for the subsequent cell wall digestion [37, 38]. We, in fact, noticed that in the case of growth medium not supplemented with sucrose and proline (the latter acts as osmo-protectant [39]), mycelium suspension into the digestion buffer led to the formation of compact tough pellets, which were difficult to convert into protoplasts. Sub-cultivation of Planobispora rosea in a starch-based medium containing sucrose and proline (Medium VM, Table 2) gave the best compromise between biomass production and protoplast yield and thus it was selected for the following experimentation.

When different combinations of commercially available lysozyme (hen egg white lysozyme or HEWL) $(5.0 \mathrm{mg} / \mathrm{ml})$ and other enzymes were tested, only the one containing mutanolysin (lysozyme from Streptomyces globisporus or ML1) led to a significant production of protoplasts (Table $3)$. It has been reported that lysozymes from diverse biological sources show a different specificity for peptidoglycan preparations from a number of Gramnegative and Gram-positive bacteria [40]. Planobispora rosea peptidoglycan was better cleaved by a Streptomyces enzyme. Addition of proteolytic enzymes was not helpful (Table 3). Optimal concentration of mutanolysin to convert mycelium to stable protoplasts was at $0.018 \mathrm{mg} / \mathrm{ml}$. A generalized positive effect on protoplast formation was observed by the addition of the non-ionic detergent Pluronic at a concentration of $100 \mathrm{mg} /$ liter. Likely, Pluronic protected protoplasts from hydrodynamic damage as already reported for eukaryotic cells [41, 42]. The efficiency of protoplast formation was assayed by microscopic enumeration at different times of incubation in the digestion solution. In contrast to the fast protoplast formation observed in Streptomyces spp. (ca.15 minutes) [43], maximum protoplast yield $\left(10^{7} / 10^{8}\right.$ protoplasts per $\mathrm{ml}$ of culture) were achieved with Planobispora rosea after 16 to 24 hours of incubation. This results in approximately $70 \%$ conversion of the mycelium to protoplasts. No appreciable difference was observed depending on whether the mycelium was sampled during the exponential or the stationary phase of growth (data not shown).

\section{Protoplast Regeneration}

Regenerating mycelium from protoplasts in Planobispora rosea has been attempted by using a series of hypertonic synthetic agar media reported in the literature $[9,19,33$, 43]. None of them supported Planobispora rosea growth. We used 1/10 diluted Medium V (Medium V0.1, Table 2) and its hypertonic version (VM0.1, Table 2) supplemented with $103 \mathrm{~g}$ /liter sucrose and $3.5 \mathrm{~g} /$ liter proline. In this last condition, regenerating colonies were observed after $15 \sim 30$ days of incubation at $30^{\circ} \mathrm{C}$. To distinguish colonies regenerated from protoplasts from those growing from residual contaminating hyphal fragments, samples from different protoplast preparations were diluted in $\mathrm{P}$ medium and then plated both on non-permissive Medium V0.1 and on permissive Medium VM0.1. Fig. 1 shows that short time incubation (1, 3, 6 hours) in protoplasting solution resulted in high contamination by whole cells (despite filtration through glass wool), since a comparable number of colonies grew in both the media. After 24-hour treatment, colonies were detectable only in hypertonic medium. In these conditions, $0.1 \%$ of the protoplasts counted by microscope regenerated to produce colonies (Fig. 1). No appreciable difference in regeneration efficiency was observed if protoplasts originated from hyphae in the exponential or in the stationary phase of growth (data not shown).

To improve the regeneration efficiency of 24-hours protoplasts, Medium VM0.1 was modified. We observed that reducing sucrose concentration from 103 to $80 \mathrm{~g} /$ /iter

Table 3 Effect of digestion enzymes combined with $5.0 \mathrm{mg} /$ liter lysozyme (HEWL) on protoplast formation

\begin{tabular}{|c|c|c|c|c|}
\hline Enzyme & $\begin{array}{l}\text { Concentration } \\
\text { range }(\mathrm{mg} / \mathrm{ml})\end{array}$ & $\begin{array}{l}\text { Protoplast } \\
\text { formation }\end{array}$ & $\begin{array}{c}\text { Number of protoplasts } \\
\text { per } \mathrm{ml} \text { in } 24 \text { hours }\end{array}$ & $\begin{array}{l}\text { Reference or } \\
\text { source }\end{array}$ \\
\hline None & - & $<10^{5}$ & & \\
\hline Mutanolysin & $0.001 \sim 25$ & ++ & $1 \times 10^{7} \sim 1 \times 10^{8}$ & Reference 44 \\
\hline Achromopeptidase & $5 \sim 25$ & - & $<10^{5}$ & Reference 9 \\
\hline Proteinase & $5 \sim 25$ & - & $<10^{5}$ & This work \\
\hline Endoprotease & $5 \sim 25$ & - & $<10^{5}$ & This work \\
\hline
\end{tabular}

-: None or scarce; ++: Good. 


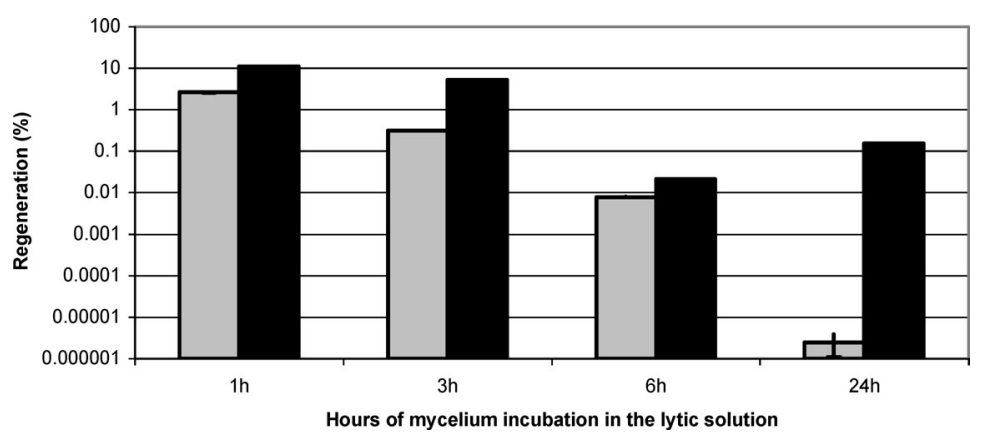

Fig. 1 Protoplast regeneration from cultures incubated for different times with digestion enzymes.

Protoplasts were plated on Medium V0.1 (grey bars) and Medium VM0.1 (black bars). Colonies growing on Medium V0.1 originated from residual hyphal fragments, while protoplasts regenerated only on Medium VM0.1. Incomplete treatment with digestion enzymes originated a high proportion of contaminating hyphae.

improved the growth rate and the dimension of regenerating colonies (data not shown), although the final number of regenerating colonies did not change. Following Okanishi and co-workers [19], regeneration medium was modified by searching for the optimal concentrations of $\mathrm{CaCl}_{2}, \mathrm{MgCl}_{2}$ and phosphate. $\mathrm{CaCl}_{2}$ addition was found to exert a positive effect, which was antagonized by phosphate buffer. $\mathrm{MgCl}_{2}$ showed a limited impact on protoplast regeneration (data not shown). The best combination of the supplemented micronutrients was $10 \mathrm{mmol} / \mathrm{liter} \mathrm{MgCl}_{2}, 50 \mathrm{mmol} / \mathrm{liter}$ $\mathrm{CaCl}_{2}$ and no phosphate. In this condition (M3 medium, Table 2) $30 \sim 50 \%$ of the protoplasts observed by microscope were regenerated and formed colonies. Previously reported efficiencies of protoplast regeneration in rare actinomycetes ranged from 1 to $37 \%$, i.e. $15 \%$ in Actinomadura verrucospora [29], 4.0\% in Micromonospora echinospora [23], 30\% in Micromonospora rosaria [21], $36.7 \%$ in Micromonospora purpurea [9], 6.6\% in Saccharopolyspora eritharea [28], and $5.0 \%$ in Amycolatopsis orientalis [24]. Protoplast regeneration efficiency was up to $90 \%$ in Streptomyces spp. [33].

\section{Recombination of Resistance Genes by Protoplast Fusion}

Protoplast fusion has been demonstrated as a powerful tool for the WGS when alternated with classical random mutagenesis in the improvement of antibiotic producing fungi and actinomycetes [16 18]. A general requirement for the assessment of this methodology is the possibility to calculate recombination frequency between genomes carrying different selectable markers. To this purpose, protoplasts of mutants of Planobispora rosea previously selected as resistant to streptomycin (162 and RS9) or gentamicin (G67 and G2) [30] (see Table 1 for strain description), were fused in the following combinations: $162 \times \mathrm{G} 2,162 \times \mathrm{G} 67, \mathrm{RS} 9 \times \mathrm{G} 2$ and RS9 $\times$ G67 (Fig. 2). As previously demonstrated the mutations selected in the mutants 162, RS9, G2 and G67 conferred resistance to the antibiotic and also determined an increase in production of GE2270 [30]. As a consequence, we expected that the combination of resistances also determined variations in the GE2270 production.

Protoplast fusion was performed according to Hopwood et al. [12] using PEG1000 as the aggregating agent. Protoplasts regenerated poorly on M3 medium supplemented with antibiotics. Thus, the efficiency of recombination between the resistant strains was determined by replica-plating regenerated colonies onto plates supplemented with streptomycin and gentamicin, streptomycin, gentamicin and on Medium V0.1 without antibiotics. The percentage of $\operatorname{str}^{\mathrm{r}} \operatorname{gen}^{\mathrm{r}}, \operatorname{str}^{\mathrm{s}} \operatorname{gen}^{\mathrm{s}}, \operatorname{str}^{\mathrm{s}} \operatorname{gen}^{\mathrm{r}}$, str $^{r} \mathrm{gen}^{\mathrm{s}}$ clones was determined. Un-fused protoplasts from the parental strains were used as controls. Fig. 2 shows that the frequency of double sensitive $\left(\operatorname{str}^{\mathrm{s}} \mathrm{gen}^{\mathrm{s}}\right.$ ) clones (which are the leading indicators of genome recombination-see below) originating from protoplast fusion ranged from 3.6 to $18 \%$ of the population, whereas the spontaneous double sensitive colonies in the un-fused populations never exceeded the $0.01 \%$. The frequency of double-resistant clones $\left(\operatorname{str}^{\mathrm{r}} \mathrm{gen}^{\mathrm{r}}\right)$ ranged instead between 8.9 and $29 \%$. Discrepancies between the population of double sensitive and double resistant clones in favour of the phenotype $\operatorname{str}^{\mathrm{r}} \mathrm{gen}{ }^{\mathrm{r}}$ was probably determined by spontaneous mutations determining $\operatorname{str}^{\mathrm{r}}$ at high frequency (as already reported for G2 and G67 strains [30]) and/or by the fact that, in myceliar microorganisms, different genomes (i.e. $\operatorname{str}^{\mathrm{r}} \mathrm{gen}^{\mathrm{s}}$ and $\operatorname{str}^{\mathrm{s}} \mathrm{gen} \mathrm{n}^{\mathrm{r}}$ ) can coexist determining a str ${ }^{r} \operatorname{gen}^{r}$ phenotype. Efficiency of protoplast fusion and genome recombination varied depending on which strains were crossed, but both the events occurred at significantly higher frequency in comparison to spontaneous mutation (Fig. 2). 


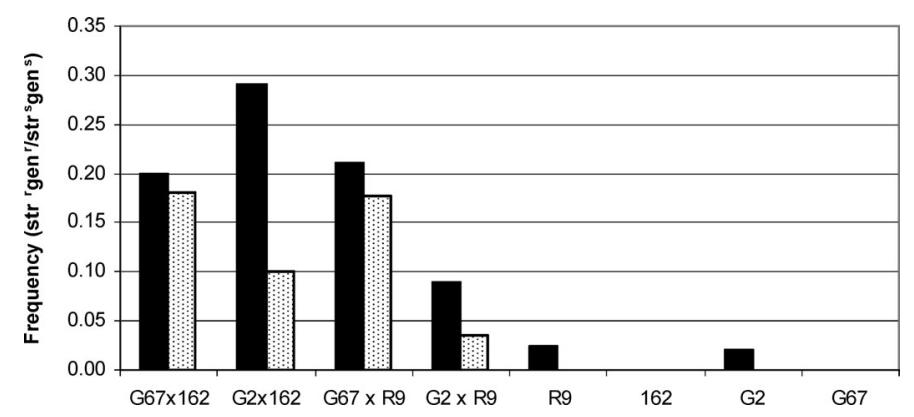

Fig. 2 Frequency of double resistance (str'gen — black bars) and double sensitive (strs gen ${ }^{\mathrm{s}}$ - dotted bars) clones originating after bi-parental protoplast fusion.

Resistant clones were selected by replica on plates supplemented with streptomycin and gentamicin. In sensitive clones the gen ${ }^{r}$ and $\mathrm{str}^{\mathrm{r}}$ of the parental clones was excluded by replica on str and gen plates. Data concerning productivity of the fusing strains are described in detail in the text.

The recombination of mutants G67×162 yielded double resistant fusants $\left(\operatorname{str}^{\mathrm{r}} \mathrm{gen}^{\mathrm{r}}\right)$ showing a GE2270 productivity (relative to the PR1/5 strain productivity [30]) intermediate between the parental strains (1.33 vs. 1.10 fold for strain 162 and 1.5 for strain G67). Similar results were obtained from the fusion $\mathrm{G} 2 \times 162$ (1.39 vs. 1.1 fold for strain 162 and 1.44 for strain G2). Double resistant fusants from recombination of G67 $\times$ RS9 were typically grouped in two classes: fusants producing less GE2270 than G67 ( $c a .1 .33$ fold) and those giving an intermediate productivity between the two parental strains (1.60 vs. 1.50 fold for strain G67 and 1.77 for strain RS9). This two classes distribution was also obtained from the G2 $\times$ RS9 fusion and it is explained by the presence in RS9 of two distinct mutations (conferring streptomycin and rifamycin resistance), whose only one was selected (the one conferring streptomycin resistance). Overall, these GE2270 production data from fused and regenerated colonies confirm our previous observations on sequentially mutated $\operatorname{str}^{\mathrm{r}} \mathrm{gen}{ }^{\mathrm{r}}$ strains showing intermediate productivity between the high producer parental strains resistant either to streptomycin or to gentamicin [30]. This proves that once proptoplasts are efficiently produced, fused and regenerated, WGS in actinomycetes may replace labour intensive procedures of recombining different mutations independently achieved by random mutation. Sister-crossing and back-crossing approaches became as feasible as in eucariotyc cells capable of sexual mating [7, 13 18].

Further work is ongoing to apply this methodology to other uncommon actinomycetes producing valuable antibiotics such as Nonomuraea, Microtetraspora, Planomonospora and Streptosporangium spp. Preliminary results show that these protocols of protoplast formation and fusion are broadly applicable to other actinomycetes, whereas the more critical and strain-specific phase is the protoplast regeneration, which requires the development of ad hoc regeneration medium (F. Beltrametti, unpublished results).

Acknowledgments We are grateful to Giancarlo Lancini for critically reviewing the manuscript.

\section{References}

1. Thiemann JE, Beretta G. A new genus of the Actinoplanaceae: Planobispora, gen. nov. Arch Mikrobiol 62: 157-166 (1968)

2. Kettenring J, Colombo L, Ferrari P, Tavecchia P, Nebuloni M, Vekey K, Gallo GG, Selva E. Antibiotic GE2270 a: a novel inhibitor of bacterial protein synthesis. II. Structure elucidation. J Antibiot 44: $702-715$ (1991)

3. Jabes D, Cavaleri M, Romanò G, Mosconi G, Bojar R, Cunliffe W. Presented at the 20th World Congress of Dermatology, Paris, July, 2002.

4. Lazzarini A, Cavaletti L, Toppo G, Marinelli F. Rare genera of actinomycetes as potential producers of new antibiotics. Antonie Van Leeuwenhoek 79: 399-405 (2001)

5. Ness JE, Welch M, Giver L, Bueno M, Cherry JR, Borchert TV, Stemmer WP, Minshull J. DNA shuffling of subgenomic sequences of subtilisin. Nat Biotechnol 17: 893-896 (1999)

6. Patnaik R, Louie S, Gavrilovic V, Perry K, Stemmer WP, Ryan CM, del Cardayre S. Genome shuffling of Lactobacillus for improved acid tolerance. Nat Biotechnol 20: 707-712 (2002)

7. Stephanopoulos G. Metabolic engineering by genome shuffling. Nat Biotechnol 20: 666-668 (2002)

8. Hopwood DA. Genetic studies with bacterial protoplasts. Annu Rev Microbiol 35: 237-272 (1981)

9. Ogawa H, Imai S, Satoh A, Kojima M. An improved method for the preparation of streptomycetes and Micromonospora protoplasts. J Antibiot 36: 184-186 (1983)

10. Baltz RH. Genetic recombination in Streptomyces fradiae by protoplast fusion and cell regeneration. J Gen Microbiol 
107: 93-102 (1978)

11. Hopwood DA, Wright HM, Bibb MJ, Cohen SN. Genetic recombination through protoplast fusion in Streptomyces. Nature 268: 171-174 (1977)

12. Hopwood DA, Wright HM. Factors affecting recombinant frequency in protoplast fusions of Streptomyces coelicolor. J Gen Microbiol 111: 137-143 (1979)

13. Kitano K, Nozaki Y, Imada A. Strain improvement of a carbapenem antibiotic producer, Streptomyces griseus subsp. cryophilus C-19393, by protoplast fusion. Agric Biol Chem 49: 685-692 (1984)

14. Wesseling AC, Lago BD. Develop Industrial Microbiol 22: 641 (1981)

15. Zhang YX, Perry K, Vinci VA, Powell K, Stemmer WP, del Cardayre SB. Genome shuffling leads to rapid phenotypic improvement in bacteria. Nature 415: 644-646 (2002)

16. Hida H, Yamada T, Yamada Y. Genome shuffling of Streptomyces sp. U121 for improved production of hydroxycitric acid. Appl Microbiol Biotechnol 73: 1387-1393 (2007)

17. Wang Y, Li Y, Pei X, Yu L, Feng Y. Genome-shuffling improved acid tolerance and L-lactic acid volumetric productivity in Lactobacillus rhamnosus. J Biotechnol 129: 510-515 (2007)

18. Petri R, Schmidt-Dannert C. Dealing with complexity: evolutionary engineering and genome shuffling. Curr Opin Biotechnol 15: 298-304 (2004)

19. Okanishi M, Suzuki K, Umezawa H. Formation and reversion of Streptomycete protoplasts: cultural condition and morphological study. J Gen Microbiol 80: 389-400 (1974)

20. Kojic M, Topisirovic L, Vasiljevic B. Efficient transformation of Micromonospora melanosporea protoplasts by Streptomyces plasmid. Curr Microbiol 23: 343-345 (1991)

21. Ryu DD, Kim KS, Cho NY, Pai HS. Genetic recombination in Micromonospora rosaria by protoplast fusion. Appl Environ Microbiol 45: 1854-1858 (1983)

22. Kim KS, Cho NY, Pai HS, Ryu DD. Mutagenesis of Micromonospora rosaria by using protoplasts and mycelial fragments. Appl Environ Microbiol 46: 689-693 (1983)

23. Love SF, Maiese WM, Rothstein DM. Conditions for protoplasting, regenerating, and transforming the calicheamicin producer, Micromonospora echinospora. Appl Environ Microbiol 58: 1376-1378 (1992)

24. Matsushima P, McHenney MA, Baltz RH. Efficient transformation of Amycolatopsis orientalis (Nocardia orientalis) protoplasts by Streptomyces plasmids. J Bacteriol 169: 2298-2300 (1987)

25. Stegmann E, Pelzer S, Wilken K, Wohlleben W. Development of three different gene cloning systems for genetic investigation of the new species Amycolatopsis japonicum MG417-CF17, the ethylenediaminedisuccinic acid producer. J Biotechnol 92: 195-204 (2001)

26. Palleroni NJ. Genetic recombination in Actinoplanes brasiliensis by protoplast fusion. Appl Environ Microbiol 45: 1865-1869 (1983)

27. Chung SK, Oh YK, Taylor P, Gerber R, Nisbet LJ. Biosynthetic studies of aridicin antibiotics. II. Microbial transformations and glycosylations by protoplasts. J Antibiot 39: 652-659 (1986)

28. Rajnisz A, Solecka J, Kurzatkowski W. Properties of Saccharopolyspora erythraea strains after protoplast regeneration. Folia Microbiol (Praha) 50: 13-18 (2005)

29. Dairi T, Hamano Y, Furumai T, Oki T. Development of a self-cloning system for Actinomadura verrucosospora and identification of polyketide synthase genes essential for production of the angucyclic antibiotic pradimicin. Appl Environ Microbiol 65: 2703-2709 (1999)

30. Beltrametti F, Rossi R, Selva E, Marinelli F. Antibiotic production improvement in the rare actinomycete Planobispora rosea by selection of mutants resistant to the Aminoglycosides Streptomycin and Gentamicin and to Rifamycin. J Ind Microbiol Biotechnol 33: 283-288. Epub 2005 Dec 2006 (2006)

31. Gastaldo L, Marinelli F. Changes in GE2270 antibiotic production in Planobispora rosea through modulation of methylation metabolism. Microbiology 149: 1523-1532 (2003)

32. Beltrametti F, Lazzarini A, Brunati C, Selva E, Marinelli F. Production of demannosyl-A40926 by a Nonomuraea sp. ATCC 39727 mutant strain. J Antibiot 56: 310-313 (2003)

33. Shirahama T, Furumai T, Okanishi M. A modified regeneration method for streptomycete protoplasts. Agric Biol Chem 45: 1271-1273 (1981)

34. Hopwood DA, Wright HM. Bacterial protoplast fusion: recombination in fused protoplasts of Streptomyces coelicolor. Mol Gen Genet 162: 307-317 (1978)

35. Siegel JL, Hurst SF, Liberman ES, Coleman SE, Bleiweis AS. Mutanolysin-induced spheroplasts of Streptococcus mutants are true protoplasts. Infect Immun 31: 808-815 (1981)

36. Hodgson DA. Primary metabolism and its control in streptomycetes: a most unusual group of bacteria. Adv Microb Physiol 42: 47-238 (2000)

37. Garcia-Dominguez M, Martin JF, Mahro B, Demain AL, Liras P. Efficient plasmid transformation of the beta-lactam producer Streptomyces clavuligerus. Appl Environ Microbiol 53: 1376-1381 (1987)

38. Hopwood DA, Chater KF, Dowding JE, Vivian A. Advances in Streptomyces coelicolor genetics. Bacteriol Rev 37: 371-405 (1973)

39. Le Rudulier D, Strom AR, Dandekar AM, Smith LT, Valentine RC. Molecular biology of osmoregulation. Science 224: 1064-1068 (1984)

40. Nakimbugwe D, Masschalck B, Deckers D, Callewaert L, Aertsen A, Michiels CW. Cell wall substrate specificity of six different lysozymes and lysozyme inhibitory activity of bacterial extracts. FEMS Microbiol Lett 259: 41-46 (2006)

41. Palomares LA, Gonzalez M, Ramirez OT. Evidence of 
Pluronic F-68 direct interaction with insect cells: impact on shear protection, recombinant protein, and baculovirus production. Enzyme Microb Technol 26: 324-331 (2000)

42. Papoutsakis ET. Media additives for protecting freely suspended animal cells against agitation and aeration damage. Trends Biotechnol 9: 316-324 (1991)
43. Kieser T, Bibb MJ, Buttner MJ, Chater KF, Hopwood DA. Practical Streptomyces Genetics: The John Innes Foundation, Norwich (2000)

44. Calandra GB, Cole RM. Lysis and protoplast formation of group B streptococci by mutanolysin. Infect Immun 28: 1033-1037 (1980) 